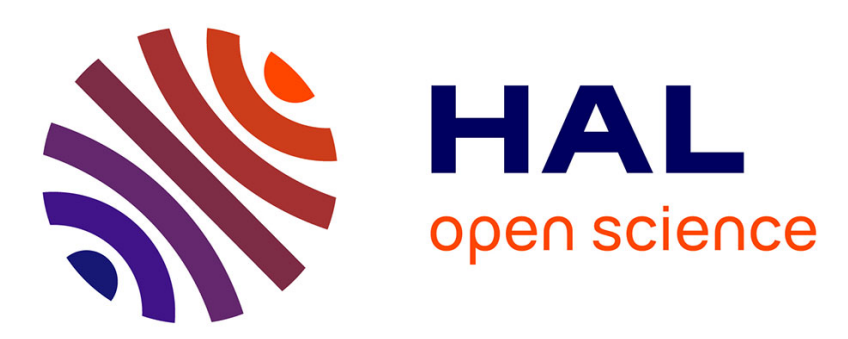

\title{
Spectromètre pour photons énergiques de faible intensité
}

A. Degré, M. Schaeffer, M. Suffert

\section{To cite this version:}

A. Degré, M. Schaeffer, M. Suffert. Spectromètre pour photons énergiques de faible intensité. Revue de Physique Appliquée, 1969, 4 (2), pp.247-248. 10.1051/rphysap:0196900402024700 . jpa-00243244

\section{HAL Id: jpa-00243244 https://hal.science/jpa-00243244}

Submitted on 1 Jan 1969

HAL is a multi-disciplinary open access archive for the deposit and dissemination of scientific research documents, whether they are published or not. The documents may come from teaching and research institutions in France or abroad, or from public or private research centers.
L'archive ouverte pluridisciplinaire HAL, est destinée au dépôt et à la diffusion de documents scientifiques de niveau recherche, publiés ou non, émanant des établissements d'enseignement et de recherche français ou étrangers, des laboratoires publics ou privés. 


\title{
SPECTROMÈTRE POUR PHOTONS ÉNERGIQUES DE FAIBLE INTENSITÉ
}

\author{
A. DEGRÉ, M. SGHAEFFER et M. SUFFERT, \\ Basses Énergies, C.R.N., Strasbourg-Cronenbourg.
}

\begin{abstract}
Résumé. - On décrit un spectromètre permettant la détection de photons d'environ $25 \mathrm{MeV}$. Il comprend un système d'anti-empilement des impulsions et un blindage efficace contre les rayons cosmiques.
\end{abstract}

Abstract. - The description of a gamma-ray spectrometer for photons of about $25 \mathrm{MeV}$ is given. The system includes antipile-up and good cosmic-ray shielding.

I. Introduction. - L'étude des réactions de capture radiative de particules multiples $\left(\mathrm{d},{ }^{3} \mathrm{He}, \alpha\right)$ dans la région d'énergie d'excitation de la résonance géante des noyaux légers $(15-30 \mathrm{MeV})$ pose un certain nombre de problèmes dus à leur faible section efficace $(\leqslant \mu b)$, à la présence de bruits de fond intenses et à l'énergie des photons à détecter $(\sim 20 \mathrm{MeV})$. En particulier, il faut réduire l'empilement des impulsions et le bruit de fond dû au rayonnement cosmique. Ces problèmes ont été partiellement résolus dans un ensemble de détection mis au point il y a quelques années [1] pour l'étude des réactions du type $\left(d, \gamma_{0}\right)$. Afin de continuer ce genre d'études en présence de bruits de fond plus intenses ou pour des sections efficaces plus faibles, il était indispensable de réaliser un ensemble plus performant. Ce nouvel ensemble est décrit dans la prochaine section et ses performances sont données dans la dernière section.

II. Description. - Le détecteur gamma est un cristal de $\mathrm{INa}(\mathrm{Tl})$ de $12,5 \mathrm{~cm}$ de diamètre et de $15 \mathrm{~cm}$ de long accouplé à un photomultiplicateur XP 1031. La résolution en énergie de cet ensemble pour ${ }^{137} \mathrm{Cs}$ est de $11 \%$. Il est entouré par un scintillateur plastique en trois parties ( $f i g$. 1) monté en anticoïncidence. L'ensemble repose sur la plate-forme d'une table tournante et permet la mesure des distributions angulaires entre $0^{\circ}$ et $160^{\circ}$.

L'électronique est assez semblable à celle de la référence [2]. Elle permet la réduction efficace de

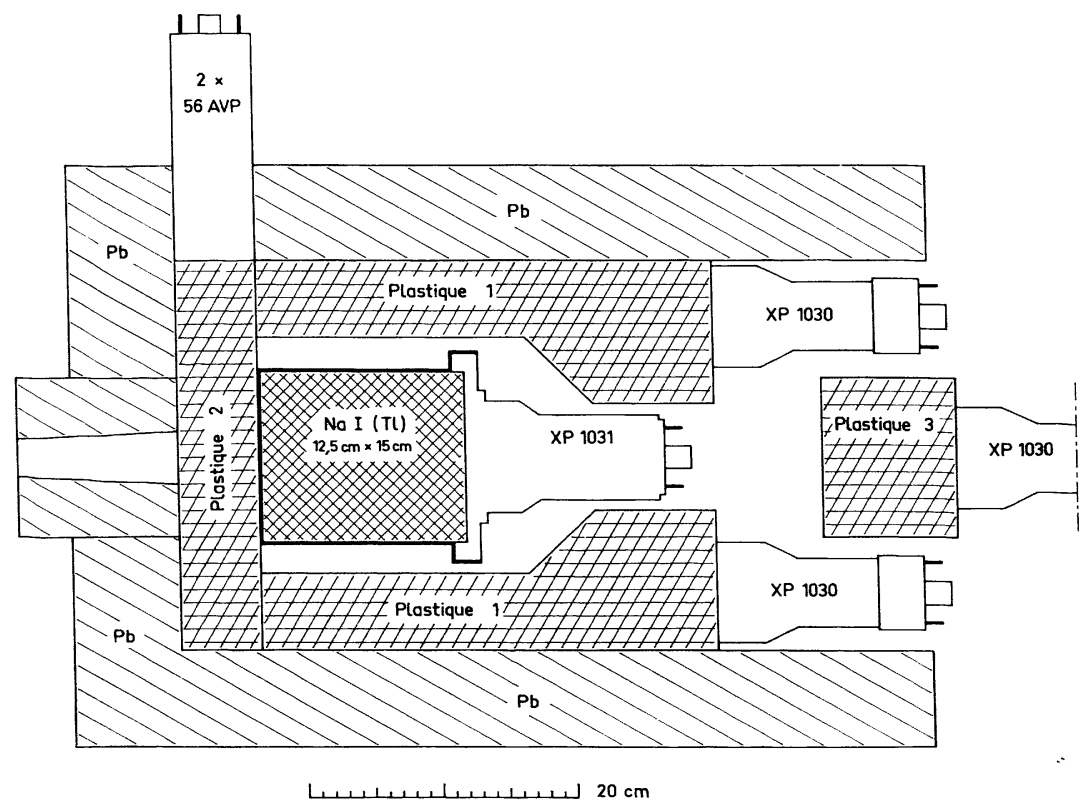

Fig. 1. - Schéma du détecteur. Le collimateur en plomb est interchangeable. 
l'empilement des impulsions à l'aide d'un discriminateur rapide. D'autre part, une anticoïncidence « très lente » $(25 \mu \mathrm{s})$ empêche l'analyse des impulsions dues à des désintégrations de muons dans INa et permet ainsi la réduction du bruit de fond cosmique résiduel.

III. Résultats. - Les différentes performances (résolution en énergie, efficacité et réjection des rayons cosmiques) dépendent de l'énergie des photons, du niveau de discrimination dans la voie d'anticoïncidence et de la collimation des photons incidents. Afin de déterminer les meilleures conditions adaptées à chaque type d'expériences, un certain nombre de combinaisons ont été testées et les résultats les plus significatifs sont donnés dans cette section ( fig. 2).

III.1. BRuit de FOND GOSMIQUe RÉSIDUel. - Le bruit de fond dû au rayonnement cosmique sans anticoïncidence est de 150 coups $/ \mathrm{MeV} / \mathrm{h}$ à $E_{\gamma}=25 \mathrm{MeV}$ et de l'ordre de 2 coups $/ \mathrm{MeV} / \mathrm{h}$ avec un niveau de discrimination moyen ( fig. 2). Un abaissement de ce niveau n'apporte pas d'amélioration dans la réjection et il faut donc supposer que le bruit de fond résiduel est dû à des composantes non ionisantes du rayonnement cosmique.

III.2. RÉSOLUTION EN ÉNERGIE ET EFFICAGITÉ. — La figure 2 donne aussi la dépendance de la résolution en énergie (largeur à mi-hauteur) et de l'efficacité en fonction du niveau de discrimination anticoïncidence, de $E_{\gamma}$ et de la collimation des photons. L'efficacité est définie comme le rapport des coups se trouvant dans une tranche de $3,12 \mathrm{MeV}$ centrée sur le pic au nombre de photons entrant dans le cristal. Cette tranche de $3,12 \mathrm{MeV}$ est celle effectivement utilisée dans l'analyse des résultats des expériences en cours. Ces différentes courbes permettent à l'utilisateur de choisir le meilleur compromis entre les différents paramètres d'après la

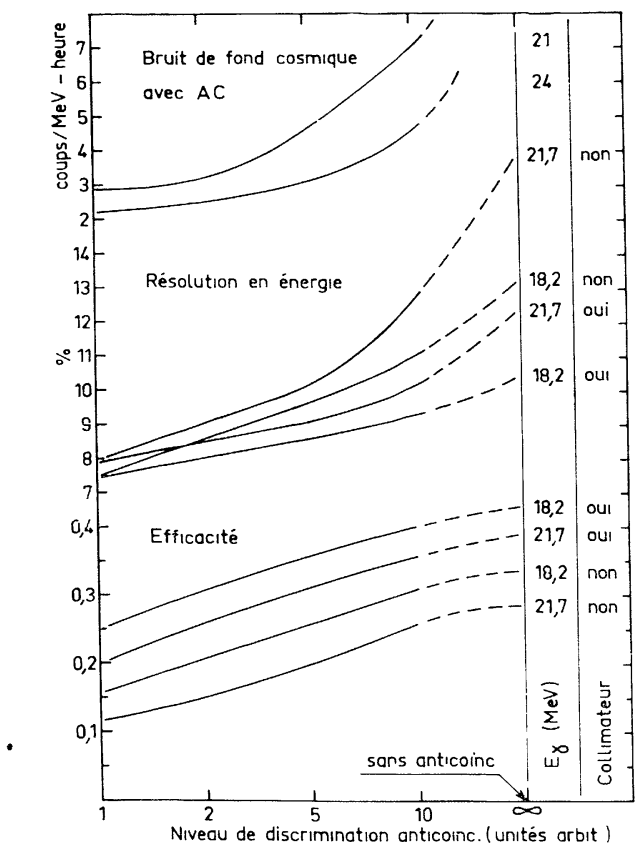

FIG. 2. - Influence du niveau de discrimination dans la voie d'anticoïncidence, de l'énergie et de la collimation des photons sur le bruit de fond cosmique résiduel, la résolution en énergie et l'efficacité. La collimation est celle de la figure 1 .

situation expérimentale. Enfin, un système semblable, mais comprenant un cristal de $\mathrm{INa}(\mathrm{Tl})$ de $25 \mathrm{~cm}$ $\times 30 \mathrm{~cm}$ est en cours de construction. Ses photomultiplicateurs et son électronique seront nettement plus rapides. Il est particulièrement destiné à travailler dans les bruits de fond intenses produits par le faisceau de particules de 10 à $20 \mathrm{MeV}$ d'un accélérateur du type Tandem.

\section{BIBLIOGRAPHIE}

[1] Suffert (M.), Nucl. Phys., 1966, 75, 226.

[2] Suffert (M.), Feldman (W.), Mahieux (J.) et Hanna (S. S.), Nucl. Instr. Methods, 1968, 63, 1. 